\title{
RECYCLING AND TRANSPORT OF NEUTRALS
}

\author{
D.Reiter \\ Institut für Energie- und Klimaforschung, Forschungszentrum Jülich GmbH, \\ EURATOM Association, Trilateral Euregio Cluster \\ D-52425 Jülich, Germany
}

\begin{abstract}
Removal of helium, the ash from the D-T-fusion reaction, from a burning plasma flame, is one of the critical issues for future thermonuclear burning plasma. Even in plasmas driven by additional heating to large Q-values this is a severe problem. Recombination of fuel and ash ions at plasma exposed surfaces, re-emission as neutral particles and subsequent pumping ("recycling") provides, at least in principle, the mechanism to flush the plasma from its ash. However, plasma surface interaction has to be limited in order to protect vessel components from excessive thermal load, often a conflicting requirement.
\end{abstract}

\section{INTRODUCTION}

This lecture deals with two, on first sight only loosely related topics. Firstly with the issue of helium removal from a stationary burning D-T fusion device, and secondly with the so called "recycling process" in the plasma near exposed first wall components. The strong interrelation of these two issues will be a major point in the following discussion.

Any kind of steady burning process depends upon both sufficient thermal insulation (to keep the temperature in the flame above a critical value) and, at the same time, sufficient particle throughput (re-fuelling, and ash removal). In the flame of a usual fire, this temperature is of the order of 1000 Kelvin, and the buoyancy driven flow of hot (used) air out of the flame provides the particle throughput. (For example a simple candle flame is choked within seconds by its own ash, if gravity is absent, as has for example been shown in demonstration experiments carried out during space flights).

For a D-T fusion plasma flame, these considerations can be translated into the terse statement, that the quality of thermal insulation (quantified by an energy confinement time) must exceed one tenth of the particle lifetime in the flame.

\section{BURN CONDITION IN THE PRESENCE OF HE- LIUM ASH}

To see this, we first consider the power balance equation, as it is often discussed for thermonuclear burn criteria (see the lecture by E.Rebhan and G. Van Oost, this issue, reference [1]). We replace the quasineutrality assumption $n_{e}=n_{i}$ (the electron density and ion density, respectively) made there now by the more general expression $n_{e}=n_{i}+2 \cdot n_{H e}$ (because the helium ion is fully ionized under reactor conditions). Introducing the fractional densities $f_{i}=n_{i} / n_{e}, f_{H e}=n_{H e} / n_{e}$ and $f_{\text {tot }}=n_{\text {tot }} / n_{e}$ for the fuel ions, the helium ash ions and the total particle content (electrons, fuel ions and ash) respectively, the equation for the fusion product $p \tau_{E_{2}}$ (loc. cit.) becomes:

$$
p \tau_{E}=\frac{\left(f_{t o t} / 2\right)^{2}}{f_{i}^{2} \frac{\langle\sigma v\rangle}{(k T)^{2}} \cdot \frac{E_{\alpha}}{24}-\frac{2}{3} C^{\prime} T^{-3 / 2}}
$$

The new factors $f_{\text {tot }}(\geq 1)$ and $f_{i}(\leq 1)$ describe the contribution of the helium ash to the total plasma pressure and the fuel dilution effects, respectively. $E_{\alpha}=3.5$ $\mathrm{MeV}$, i.e., we assume complete thermalization of the helium ion, before it is lost, and $\mathrm{C}$ is a constant in the expression for radiation losses, which, in this particular form of ignition condition, have not been included in the definition of $\tau_{E}$.

A similar consideration, balancing the helium particle production rate with the losses of helium particles due to their finite lifetime in the system $\left(\tau_{\alpha}^{*}\right)$, yields $([2])$

$$
p \tau_{E}=\frac{f_{H e} \cdot\left(f_{t o t} / 2\right)}{\rho \cdot f_{i}^{2} \frac{\langle\sigma v\rangle}{k T} \cdot \frac{1}{8}} \quad\left(\text { from } \frac{n_{H e}}{\tau_{\alpha}^{*}}=\frac{1}{4} n_{i}^{2}\langle\sigma v\rangle\right)
$$

$\rho$ denotes, as in [2], the confinement time ratio $\tau_{\alpha}^{*} / \tau_{E}$. We will, further on, take this ratio as a constant parameter, i.e., independent of density and temperature. One might relax that choice, e.g. by employing empirical scaling laws for $\tau_{E}$ and $\tau_{\alpha}^{*}$. But this might be already well beyond the power of such simple zero-dimensional considerations, in particular due to the possibly very 


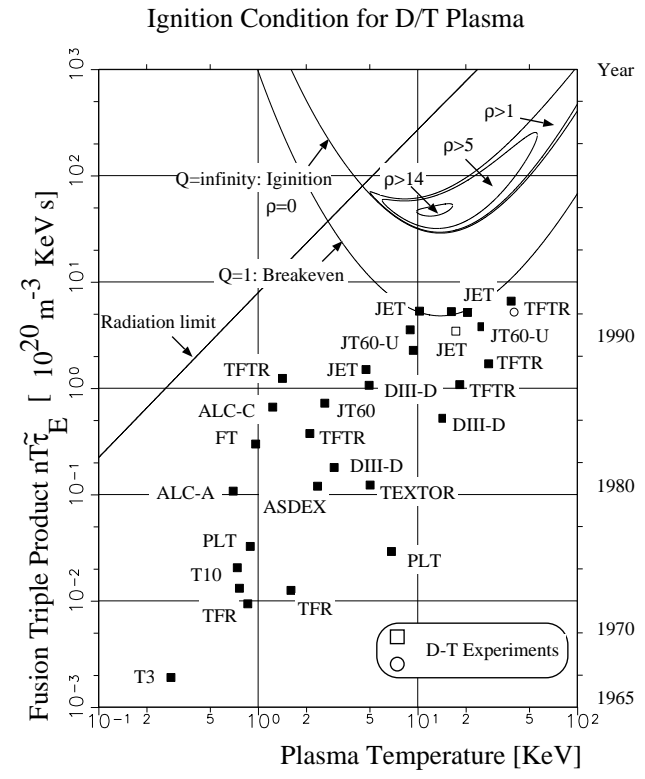

Figure 1: Fusion triple product vs. plasma temperature. Experiments, break-even $(\mathrm{Q}=1)$ and boundaries for ignition. Parameter $\rho=\tau_{\alpha}^{*} / \tau_{E}=0,1,5,14$

different spatial distributions of energy sources and recycling particle sources (see below).

Expressing $f_{i}$ and $f_{\text {tot }}$ in terms of $f_{H e}$, and equating the expressions for $p \tau_{E}$ from Eqs. (1) and (2), one arrives at a cubic algebraic equation for $f_{H e}: g\left(f_{H e}\right)=0$. The four coefficients are functions of temperature and $\rho$. Including one further free parameter $f_{Z}$ for a fractional density of impurities of charge $\mathrm{Z}$ (which contaminate the plasma due to wall erosion processes or are introduced on purpose for plasma edge temperature control) is straightforward (loc.cit.). See again refs. [1] and [2] and Figure 2.

Clearly, $f_{H e}$ must lie in the interval $(0,0.5)$.

Inspecting the cubic polynomial for $f_{H e}$, one finds one (unphysical) root outside this range, and two other roots. These are negative at temperatures significantly below the critical ignition temperature and they turn conjugate complex with increasing temperature near the critical temperature. In these two regions no steady self-sustaining plasma burn is possible. The two roots become positive then at larger temperatures, in the region between 5 and $100 \mathrm{KeV}$ (depending upon the value of $\rho$ ) and they lie in the physically accessible range between 0 and 0.5. Beyond this region the two roots turn complex again, due to the fact that radiation losses and fuel dilution prohibit steady burning at these even higher plasma temperatures.
If one inserts the physically relevant fractional helium densities obtained in this way (the algebraic closed form expressions for the roots of cubic polynomials) in either Eq. (1) or Eq. (2), the closed burn contours shown in Figure 1 (labelled $\rho=$ const) result. Note that for convenience the ordinate has been re-scaled from $n T \tau_{E}$ to $n T \tilde{\tau}_{E}$, with $\tilde{\tau}_{E}$ denoting the "global confinement time", which, in contrast to $\tau_{E}$ also contains radiation losses. In the definition of the parameter $\rho$, however, we have retained the energy confinement time $\tau_{E}$. Otherwise the second (upper) branch would disappear, and the more familiar open burn-curves would result. Whether or not the ignition curves are open or closed (one or two solutions for $f_{H e}$ ) depends upon the definition of $\rho$, not upon the definition of $\tau_{E}$ in the ordinate (e.g. in the fusion triple product).

If one uses the global confinement time $\tilde{\tau}_{E}$ to derive ignition conditions, (as it seems to be common practise) rather than $\tau_{E}$ as defined in Equation (1), and if one excludes the helium ash from consideration (i.e., if one sets $\rho=0$ ) then one has to add a further constraint (called "radiation limit", see Figure 1) to prevent unphysical ignition parameters $n T \tau_{E}$ resulting from negative transport losses (i.e., gains) balancing radiation losses.

The fact that the closed contours shrink in size and disappear beyond a certain critical ratio $\rho_{\text {crit }}\left(\rho_{\text {crit }} \approx\right.$ 15 for a pure D-T plasma) translates into the statements made above concerning the often conflicting requirements of good thermal insulation and poor particle confinement.

If one specifies a fixed relative impurity concentration $f_{Z}=n_{Z} / n_{e}$ for one (effective) impurity of charge $\mathrm{Z}$ and modifies $f_{\text {tot }}$ and the radiation term in Equation (1) accordingly, then one finds that the contours shrink in size even faster with increasing $\mathrm{Z}$ and $f_{Z}$. For each confinement parameter $\rho$ a maximum tolerable impurity concentration results, and, vice versa, for each impurity concentration one finds a critical maximum $\rho=\rho_{\text {crit }}\left(Z, f_{Z}\right)$. See Figure 2 and note the difference for light and heavy impurities (the abscissa has a logscale).

Note that the need for poorer particle confinement (i.e., larger particle fluxes onto limiter and divertor targets, hence smaller values of $\rho$ ) may result in incomplete thermalization of the supra-thermal $3.5 \mathrm{MeV}$ helium ash (we have assumed complete thermalization in the discussions above) and/or in increased surface erosion and hence impurity concentration. Whether nature provides an operational window to fulfill both requirements in an economic fusion power plant is still an open question.

Various further aspects may readily be included in this simple "point reactor analysis" without changing 


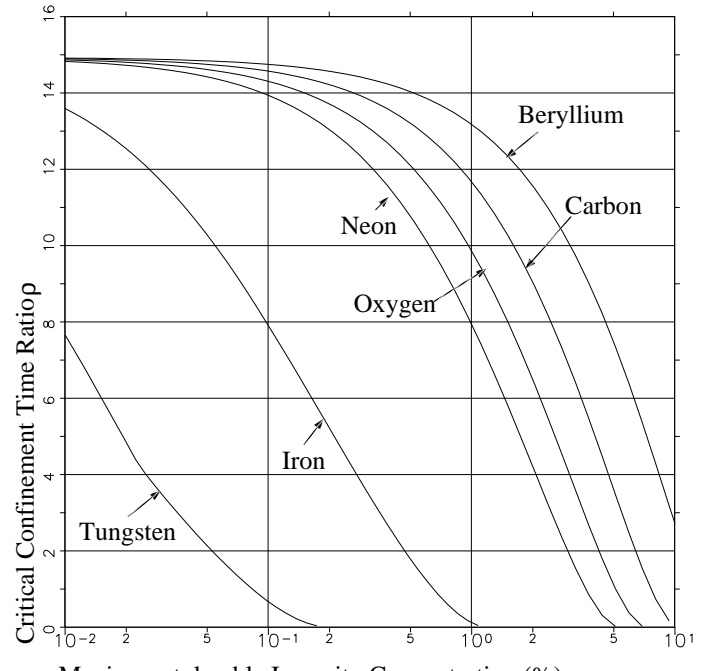

Maximum tolerable Impurity Concentration (\%)

Figure 2: Critical confinement time ratio versus maximum tolerable impurity concentration, for Beryllium, Carbon, Neon, Oxygen, Iron and Tungsten (note: logscale on abscissa)

the qualitative picture. For example the assumption of flat temperature and density profiles may be weakened by introducing profile shape factors in the balance Eqs. (1) and (2) (see e.g. ref. [3]). So called "advanced fuel" reactors based upon different fusion processes (such as D-D fusion, D- ${ }^{3} \mathrm{He}$ fusion, even including secondary fusion reactions between fusion products and the fuel particles) can be studied within the same framework.

In the case of more than one type of fusion product (say $m$ different types, e.g. ${ }^{4} \mathrm{He},{ }^{3} \mathrm{He}$, and p, and even if all parasitic fusion processes are accounted for) simply one particle balance equation for each type of ash particle has to be written. This results in a coupled set of $m$ cubic polynomials for the $m$ fractional ash densities, and again in the same type of closed ignition contours. For the cases studied so far, the critical confinement time in advanced fusion concepts ratio was found to be even smaller than in case of the D-T fusion reactor (ref. $[2])$.

\section{THE PARTICLE LIFETIME $\tau_{\alpha}^{*}$}

The energy confinement time $\tau_{E}$ in tokamak plasmas is an experimentally well characterized quantity. Various empirical scaling laws derived from large databases exist. Much less well defined is the particle confinement time $\tau_{\alpha}^{*}$ entering the above formulae. A simple relation between $\tau_{E}$ and the heat diffusivity $\chi$ is frequently used: $\tau_{E} \propto \frac{1}{\chi}$. In a similar manner the lifetime of an ash particle, born in the burning core of a fusion plasma and with a spatial source profile identical to the fusion energy source profile, can be related to a particle diffusion coefficient: $\tau_{\alpha 1} \propto \frac{1}{D}$. Both confinement times should also scale with $a^{2}$ ( $a$ denoting the small plasma radius).

This can be seen as follows:

If one assumes the particle and power input on axis, no inward pinches, spatially constant diffusivities D and $\chi$, then one finds triangular profiles, from integrating

$$
\Gamma=D d n / d r, \quad Q=n(0) \chi d T / d r
$$

and consequently

$$
T(r) \propto \frac{a}{\chi}(1-r / a), \quad n(r) \propto \frac{a}{D}(1-r / a) .
$$

This is based upon the assumption that the dominant plasma particle source (not the helium source) is still located in the edge plasma (not in the core) and hence convected power flux is negligible for most of the plasma region.

Hence the resulting confinement time ratio $\rho$ would be determined (excluding convective fluxes) by the ratio $\frac{\chi}{D}$.

Since one can expect $\tau_{\alpha 1}$ to scale with $a^{2}$ but a much weaker dependence of $\tau_{E}$ on $a$ is found experimentally, this would lead to extremely pessimistic predictions for the larger future fusion devices.

When a plasma particle finally reaches a wall, it is neutralized there and re-emitted into the plasma as neutral atom or molecule.

A fraction $\epsilon$ may be pumped away, the remaining fraction $R=1-\epsilon$ will be re-ionized in the plasma. $R$ is generally referred to as "recycling coefficient", $\epsilon$ is the pumping efficiency.

If the spatial distribution of the primary source helium ions (i.e., of the fusion alpha particles) and of the recycling source (i.e., of the re-ionized helium particles) would be approximately the same, then, as a result of non-perfect pumping, the particle confinement time $\tau_{\alpha 1}$ would simply be enhanced by the factor $\frac{1}{1-R}$.

$$
\tau_{\alpha}^{*}=\frac{\tau_{\alpha}}{(1-R)}
$$

with typical values of $\mathrm{R}$ close to one. Hence: again very pessimistic prospects.

Fortunately for the helium ash (as for the fuel particles) this similarity of source profiles for energy and particles is not the case, and a slightly more detailed consideration is necessary, carefully trying not to "overcharge" our simple model. Strictly: only transport analysis codes can provide a somewhat realistic picture. A modified expression for $\tau_{\alpha}^{*}$

$$
\tau_{\alpha}^{*}=\tau_{\alpha 1}+\frac{R}{1-R} \tau_{\alpha 2}
$$


has been derived in [2] from an analytical solution of a somewhat more refined (as compared to Eq. (2) above) but still 0-dimensional particle conservation equation.

We may consider re-ionization of recycling helium particles as a second source. This source, however, is located in the edge. Let us assume that all neutral helium particles are ionized at $r=a-\lambda_{i z}$. Hence:

$$
-D d n / d r=0 \text { for } r<a-\lambda_{i z}
$$

i.e., $\mathrm{n}(\mathrm{r})=\mathrm{n}(0)=$ constant in the core region for this contribution and $n$ decreases linearly in the ionization zone (the boundary plasma)

$$
a-\lambda_{i z}<r<a \text {. }
$$

(If a pinch $v_{\text {pinch }}=-2 D r / a^{2}$ is included, then a Gaussian $\mathrm{n}(\mathrm{r})$ profile is added on top of the densityplateau).

For this recycling source contribution one then finds a particle confinement time

$$
\tau_{\alpha 2}=\frac{a \lambda_{i z}}{D}
$$

This $\tau_{\alpha 2}$, in contrast to $\tau_{E}$ and $\tau_{\alpha 1}$, is, essentially, a plasma edge quantity, since the ionization length depends upon $T_{e}$ and $n_{e}$ in the edge, and only the value of $\mathrm{D}$ (if it is not radially constant) within the ionization zone $a-\lambda_{i z}<r<a$ matters. Inserting for the ionization length $\lambda_{i z}$

$$
\lambda_{i z} \approx \frac{v_{0}}{0.5 n_{e}<\sigma v>_{i z}}
$$

with $v_{0} \approx$ some $10^{4} \mathrm{~m} / \mathrm{s}$ the radial velocity of recycling atoms, we see that $\tau_{\alpha 2} \propto n_{e}^{-1}$. This scaling is often found in limiter-tokamaks, but not in divertortokamaks (see the lectures on edge plasma physics, and on divertors). In divertor experiments a significant fraction of re-ionization takes place within the scrape off layer, and that wrecks (amongst others) any simple relation between $n_{e}$, the flux $\Gamma$, the edge plasma density and particle confinement times $\tau_{p}$.

We may conclude, that the decisive confinement parameter $\rho$ is given as:

$$
\rho=\rho_{\text {core }}+C_{\text {edge }} / n_{e}
$$

One may not be able to do much about the core plasma transport parameter $\rho_{\text {core }}$, in particular size may not help. However, $C_{\text {edge }}$ can probably strongly be influenced by appropriate divertor or limiter design. This is the second reason, in addition to the target surface loading problem (loc. cit.), why plasma edge physics has gained so much relevance in fusion research in the last few years.

\section{A RANDOM WALK MODEL FOR $N_{H E}$}

The same result as in Equation (6) can be obtained using stochastic arguments: We consider a random walk (of the helium particle) in a system of only two states: the plasma core (birth point of the particle by fusion reaction), P1 and the limiter or divertor target, P2. All particles start at point $\mathrm{P} 1$, and travel (with probability one) to point $\mathrm{P} 2$, in a time $\tau_{\alpha 1}$. At point $\mathrm{P} 2$ they are either absorbed with probability $\epsilon$ (instantaneous transition into a final "limbo" state P3, if one wishes to look at it that way). With probability (1- $\epsilon$ ) the particle returns to $\mathrm{P} 2$. This single return trip takes $\tau_{\alpha 2}$ seconds, the lifetime of recycled particles in the plasma core.

This recycling lifetime $\tau_{\alpha 2}$ should scale weaker with the plasma size a than $\tau_{\alpha 1}$, but instead scale with the relative re-penetration depth for neutral particles. Thus: the more relevant this second time $\tau_{\alpha 2}$ for the overall mean particle lifetime $\tau_{\alpha}^{*}$, the less severe becomes the helium removal problem for larger fusion devices.

The mean lifetime of the random walking particle is given as:

$$
\tau_{\alpha}^{*}=\sum_{i=0}^{\infty} \tau_{i} \cdot p_{i},
$$

where $p_{i}$ is the probability of exactly i recycling events before pumping, and $\tau_{i}$ is time spend in the core by a particle, which is absorbed after precisely i recycling events. Clearly: $p_{i}=\epsilon \cdot(1-\epsilon)^{i}$, and $\tau_{i}=\tau_{\alpha 1}+i \cdot \tau_{\alpha 2}$. The infinite series can readily be shown to converge to:

$$
\begin{aligned}
\tau_{\alpha}^{*} & =\tau_{\alpha 1}+\frac{1-\epsilon}{\epsilon} \cdot \tau_{\alpha 2} \\
& =\tau_{\alpha 1}+\frac{R}{1-R} \tau_{\alpha 2}
\end{aligned}
$$

Rather than evaluating the infinite series equation (11) analytically, one could instead have used a pocket calculator with a random number generator. Generating a few thousand histories, each starting in point P1 and terminating in point P3 and accumulating the mean lifetime of the "test particles" would confirm (then only within statistical precision) the relation (12) above.

From this and Equation (2) we note, that the density of particles in a certain volume is given by the mean lifetime spend in that volume by random walking particles, multiplied by the source strength and divided by the volume. This stochastic procedure becomes far more handy than the analytical arguments from ref. [2], if more details are to be considered. For example the number of states can be increased to simulate the effects of several pumping stations with different pumping rates each (e.g., the effect of un-pumped divertor legs, etc.). Or the volume of interest can be subdivided 


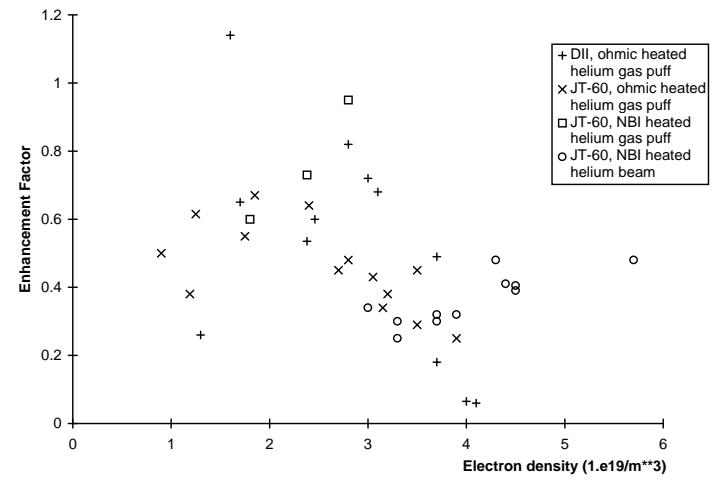

Figure 3: Helium enrichment near pumping stations from various Tokamak experiments, showing both (small) enrichment in some cases but also significant (unfavorable) de-enrichment.

into smaller volumes and the averaged density in each such cell can be computed.

We will return to this point later, but then for a very detailed random walk model with the number of possible states increased to infinity. The ChapmanKolmogoroff equation of the resulting Markoff process in that case will directly be related to the linear transport equation (in integral form) for recycling neutral particles. But the method for obtaining particle densities from estimating the mean time spend by random walkers in specified volumes will essentially remain the same.

In closing this section we note that the $\epsilon$ in Equation (12) is the (effective) probability for a particle to be pumped, once it leaves the burning plasma core. One can (see again ref. [2]) reformulate Equation (12) in terms of a true surface pumping efficiency $\epsilon_{r}$ and a screening efficiency $\epsilon_{S}$ of the plasma edge region between wall and burning core. $\gamma_{S}=1-\epsilon_{S}$ is then the probability for an un-pumped particle to return to the core before it hits the wall for the next time.

One finds:

$$
\tau_{\alpha}^{*}=\tau_{\alpha 1}+\frac{\left(1-\epsilon_{S}\right)\left(1-\epsilon_{r}\right)}{\epsilon_{r}} \tau_{\alpha 2} \approx \tau_{\alpha 1}+\frac{\gamma_{S}}{\epsilon_{S}} \tau_{\alpha 2}
$$

The decisive factor $\frac{\gamma_{S}}{\epsilon_{S}}$ describes the recycling process and is often the ratio of two small numbers. It is hard to estimate, and has to be investigated on a case to case basis, usually resorting to complex Monte Carlo simulation models.

In particular the issue of "helium enrichment" near pumping stations (i.e. the relative decrease or increase of the fractional helium pressure near pumps due to configurational and/or atomic physics effects) remains rather unpredictable for this reason. No clear trends on whether the relative abundance of helium increases or decreases in divertors or near limiters, as compared to upstream conditions, are found experimentally either. This depends upon configurational details, in particular upon the position of the pump relative to the strike point and the plasma. See Figure 3.

\section{EXPERIMENTAL RESULTS}

Since the particle lifetime $\tau_{\alpha}^{*}$ has two components with a different scaling on machine size, it is rather difficult to extrapolate from the present database to next generation fusion devices such as ITER or even to a reactor. However, keeping in mind that roughly $\rho \approx \frac{\frac{\chi}{D}}{1-R}$ in present experiments might still characterize global confinement, one may get an idea of the experimental situation firstly by assuming a realistic value of $R$, (say: $R=0.9$ ), and secondly by inspecting experimental data for the ratio $\frac{\chi}{D}$ (see [4], and references therein). Heat and density pulse propagation analysis (after sawtooth crashes) have shown $5<\frac{\chi}{D}<12$ in JET, and $\frac{\chi}{D} \approx 3-6$ in JT-60. Smaller values of $\frac{\chi}{D} \approx 2-3$ have been reported from JET from profile analysis, and $\frac{\chi}{D} \approx 4$ for L-mode and $\frac{\chi}{D} \approx 1$ for super-shot conditions in TFTR. A ratio of 5 is predicted from theory for "advanced stellarator" concepts.

Note that in case of an inward pinch, i.e. a nondiffusive inward flow of particles, the confinement time ratio is underestimated by $\frac{\chi}{D}$ even in case of perfect pumping $R=0$. The existence of such pinch effects is often indirectly concluded from the experimentally observed peaking of density profiles even inside the region of particle sources. In this source free region and for roughly constant diffusion coefficients $\mathrm{D}$, the profiles would have to be flat otherwise.

Direct experimental results for $\frac{\tau_{\alpha 1}}{\tau_{E}}$ are reported from TEXTOR and TFTR, with values in the range $\sim 2-3$ in both cases. Together with about $10 \%$ particle removal efficiency provided by the ALT-II pump-limiter at TEXTOR, the critical confinement time ratio mentioned above is presently just marginally within reach in medium sized tokamak experiments. This and taking the arguments from the previous section into account may indeed provide some optimism with regard to the ash removal issue. 


\section{RECYCLING}

As illustrated above, the mechanism of recycling of neutral particles back into the plasma plays a crucial role, and, since ratios of small numbers are involved, seemingly small details of this process can have large effects. Experimentally the neutral particle densities are found to be rather low in tokamak plasma edge regions, of the order $10^{8}$ to $10^{12}\left(\# / \mathrm{cm}^{3}\right)$. They are negligible further into the discharge, at least for the present tokamak generation such as TEXTOR, ASDEX-Upgrade and all larger devices. The only exception, occasionally, is a small region of highly localized recycling in the vicinity of the limiter strike point or near the "footprint" of the plasma on a divertor target (neutral particle densities of up to $10^{14}\left(\# / \mathrm{cm}^{3}\right)$ there).

Neutral molecules dissociate, usually in an even narrower layer at that location in the plasma where the electron temperature reaches the dissociation threshold energy (10 to $15 \mathrm{eV})$.

Neutral Franck-Condon atoms are formed there, with typically a few eV kinetic energy. Together with the other atoms, which are directly reflected from the surfaces, they penetrate the plasma.

The three figures in the right column show typical trajectories of neutral particles recycled at the divertor targets of ASDEX-Upgrade, JET and ITER divertor targets. In the first two cases they fill the divertor plasma (a smaller fraction in case of the JET divertor) and the vacuum region, but do not significantly (not at all in case of JET) penetrate the plasma core. The similar picture for ITER typical conditions shows an effective screening already of the divertor plasma against neutral particle penetration. (This causes one of the major uncertainties with regard to the ability of neutral particles to disperse power and momentum from an ITER-sized divertor in a "high recycling" regime, and has led to a revision of divertor concepts, away from high recycling divertors towards "detached" or "gas-target" divertors, see, again, the lectures on edge physics and divertors).

The trajectories in these figures are computed from a particle simulation program using the plasma density and temperature as input, and simulating the various elementary collision processes between neutral particles, the surface structures and the plasma electrons and ions.

The most dominant collision process considered here is charge exchange $(\mathrm{CX})$ : in the resonant case the neutral atom and the plasma ion exchange identity (scattering angle $\theta=\pi$ in the center of mass frame). As pointed out first by Sacharov back in 1961, it is in this way that low energy neutrals near the wall can gain energy through frequent CX scattering and penetrate
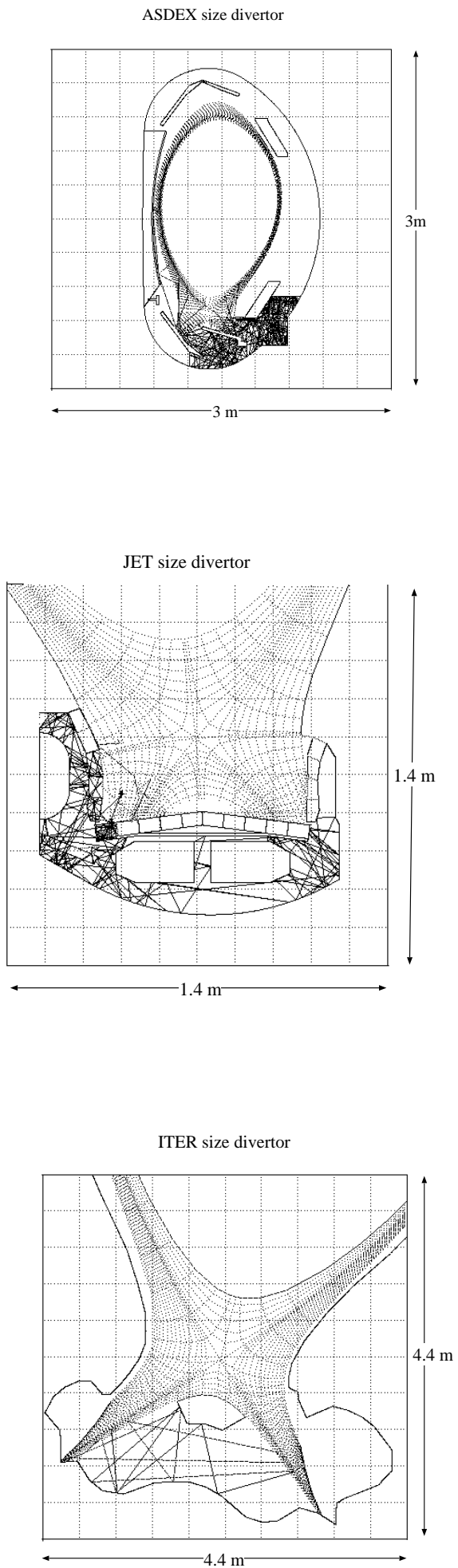

Figure 4: Neutral particle trajectories in Divertors, showing reduced penetration (compared to size) in larger divertors. 


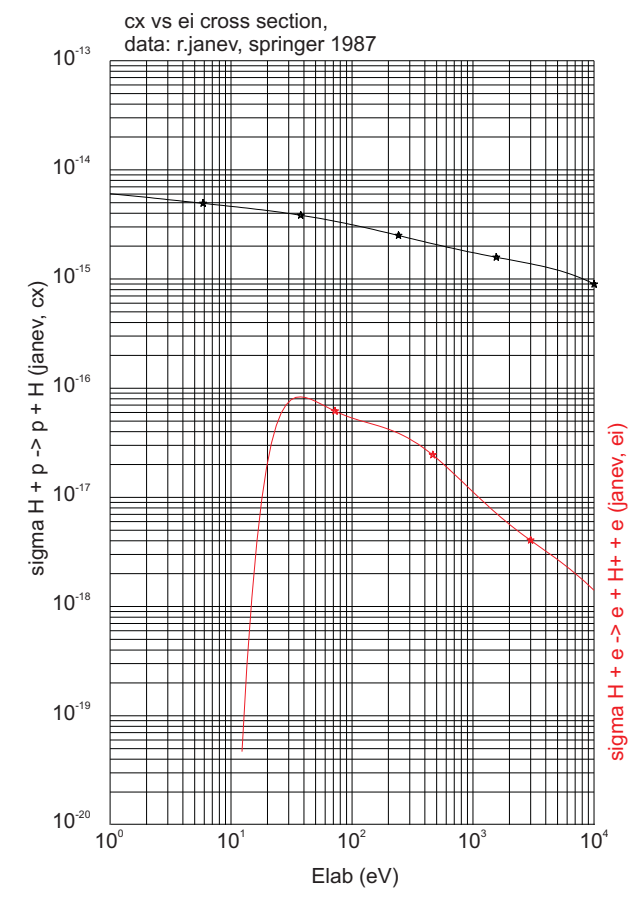

Figure 5: charge exchange cross section for hydrogen, in $\mathrm{cm}^{2}$ (upper curve) and electron impact ionization cross section (lower curve)

into the plasma interior. Typically, in present limitertokamaks the cloud of neutral atoms reaches about 10 $\mathrm{cm}$ radially into the discharge. Using simple $1 \mathrm{D}$ diffusion equations, a diffusion coefficient for neutral atom penetration $D_{n}=(\delta x)^{2} / \delta t$ with $\delta x$ the mean free path for CX and $\delta t$ the mean time between CX events, one finds an effective penetration length for neutral particles into the plasma given as the harmonic mean of the charge exchange- and the ionization mean free path, both taken at the ion thermal speed. This is far in excess of the ionization mean free path alone, taken at a speed of thermal wall atoms or $3 \mathrm{eV}$ Franck-Condon dissociation products.

This dominant resonant charge exchange (i.e.: $H+$ $p \rightarrow p+H)$ happens if the two particles, $p$ and $H$, approach each other, then, for a short time, the one electron belongs to both (an intermediate $\mathrm{H}_{2}^{+}$molecule is formed) and, after separation, the electron stays with the former ion, and leaves the former neutral now in the ionized state. Since collision partners have changed their internal energy, this would be an in-elastic process. However, quantum-mechanically, it cannot be distinguished from ordinary elastic scattering between the two partners, ie., from the event in which the electron happens to stay with the same particle after the event.

Therefore, quantum-mechanically, there is only one process, usually termed "elastic", but including both types of "events". One must carefully avoid double counting charge exchange. I.e., one must never add an quantum mechanically derived "elastic" collision rate to a charge exchange rate taken from another source. The reader can find bad examples of neutral gas transport calculations, with this severe error in the dominant reaction rates, even in a recent issue of the "Journal of Nuclear Fusion" from the year 2000 (despite a correct treatment of this issue since about 40 years in most applications in fusion research). Needless to mention: these authors find "much better agreement with experiment" after having included, erroneously, the "new elastic effect"....

The terminating event in the lifetime of a neutral particle may either be the entry into a pump, or reionization by electron impact collisions.

Usually this re-ionization process is a step-process, with various intermediate excitations, radiative decays, etc. of the neutral atom before ionization. These processes are most conveniently described in terms of so called collisional-radiative ionization models, see, e.g., the "atomic and molecular data section" in [8]. They lead to an enhancement of the mean energy dissipated from the electrons from $13.6 \mathrm{eV}$ per ionization to typically $25 \mathrm{eV}$ per ionization and even larger values at electron temperatures below $20 \mathrm{eV}$. Similar concepts apply for dissociation of molecules, dissociative recombination of molecular ions, etc... For $H_{2}$ molecules, colliding with protons, there is also a ("non-diagonal") charge exchange process, which can be resonant if the molecule is sufficiently high vibrationally excited. This leads to an atom and $\mathrm{H}_{2}^{+}$. If this molecular ion then decays (after electron impact) into two ground state atoms, this entire chain of events is, effectively, a recombination (accompanied by a dissociation of the molecule), and is therefore referred to as MAR (molecular assisted recombination). If the final products of the $\mathrm{H}_{2}^{+}$are one atom and one ion, however, then, effectively, this entire chain starting from the charge exchange is nothing but one effective dissociation (then termed MAD, molecular assisted dissociation, by abuse of language).

The competing rate coefficients for these two reaction chains are shown in Fig. 6. These coefficients must be multiplied by the local neutral molecule density and the proton density to turn them into a volumetric rate.

Clearly, for increasing plasma density, the dissociative channel grossly outweighs the recombinative channel. Despite the fact that the MAR rate may be large by itself (this having triggered quite optimistic predictions for the operational window for detached divertors initially, see e.g., the ITER physics basis studies, Nuclear Fusion, special issue, Dec. 2000) it has turned out to be a fairly irrelevant recombination process, after a more detailed computer modelling of dense edge 


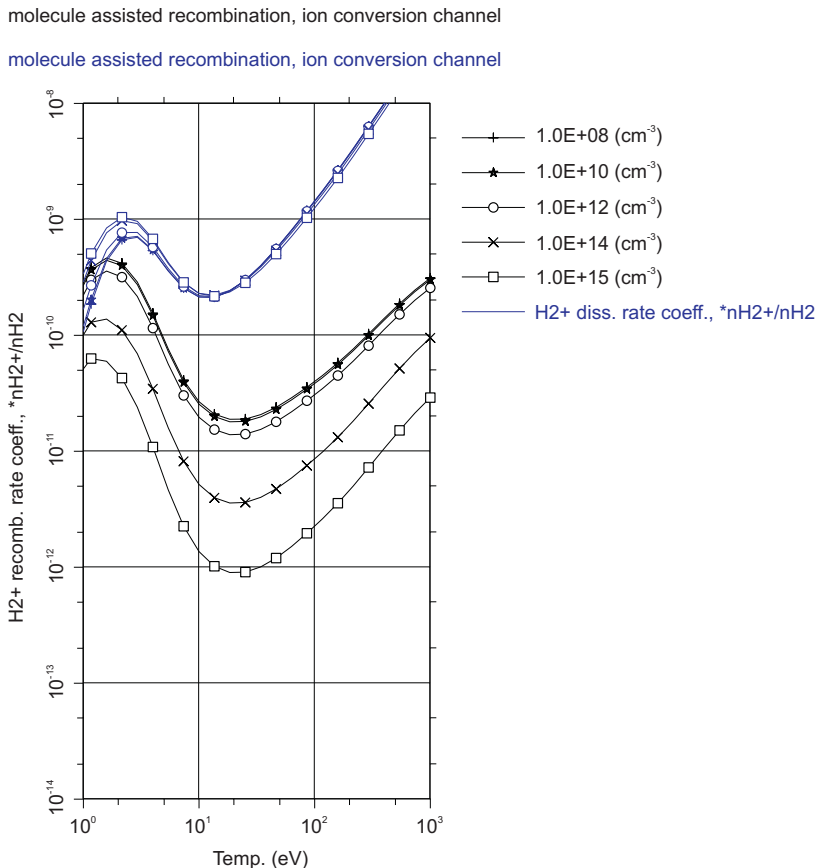

Figure 6: Rate coefficients for destruction of $\mathrm{H}_{2}$ by proton impact leading to three neutral atoms (lower set of curves, MAR), and leading to one ion and two atoms (upper set of curves, MAD). The curves are labelled by the electron density.

plasma conditions (e.g. detached divertors) had been carried out. This is because the competitive process (MAD) is even much stronger and molecules are dissociated long before they can "assist recombination", in current fusion edge plasma conditions.

In general: neutral particle recycling (atoms or molecules) is of crucial importance for the present concepts of plasma edge control: the neutral particles must be kept in the discharge long enough (i.e. the pump must not be too strong) to provide significant plasma cooling: presently 10 to $20 \%$ of the total power flowing into the edge plasma is dissipated via the neutral particle channel in high density divertor experiments.

This fraction must, according to more recent design considerations, be further increased for save reactor divertor concepts. On first sight this seems to conflict directly with the earlier discussed requirements of strong pumping and efficient particle removal. An intensive search for possible solutions out of this dilemma is presently carried out in many laboratories in the world, largely based on computer simulation experiments.

With regard to such computer models for the recycling process in particular (see again: ref. [8]) one has to note that the ratio of the charge exchange rate to the ionization rate is larger than one (typically three to five, depending upon the electron temperature and only very weakly on the ion temperature), but not very large. The neutral gas cloud is re-ionized after a few CX mean free paths into the plasma. Furthermore the density in this cloud is usually too small in order to permit neutral-neutral elastic collisions to contribute significantly to the entropy production for the neutral component. A more precise quantification of this statement is provided by a "generalized Knudsen number" for the neutral particles, which relates entropy producing processes (resonant charge exchange with Maxwellian background ions, elastic collisions) to characteristic free flight and absorption lengths:

$$
\overline{K n}_{e f f}(\vec{r})=\frac{\bar{\lambda}_{s c}(\vec{r})}{\bar{l}_{e f f}(\vec{r})}
$$

with

$$
\bar{\lambda}_{s c}(\vec{r})=\frac{1}{n_{0}(\vec{r})} \int d \vec{v} \lambda_{s c}(\vec{v}, \vec{r}) f_{0}(\vec{v}, \vec{r})
$$

and

$$
\bar{l}_{\text {eff }}=\frac{\bar{\lambda}_{\text {absorption }} \cdot \bar{l}_{\text {freeflight }}}{\bar{\lambda}_{\text {absorption }}+\bar{l}_{\text {freeflight }}}
$$

Here $f_{0}$ denotes the neutral particle distribution function, $\lambda_{s c}$ the mean free path for scattering (charge exchange and elastic), $\bar{\lambda}_{\text {absorbtion }}$ is the mean free path for ionization (averaged over $f_{0}$ similarly), and $\bar{l}_{\text {freeflight }}$ is the averaged and also spatially dependent distance to the nearest boundary. Values of $K n_{\text {eff }}$ below 0.1 indicate fluid like (diffusive) behavior of neutral particles. Unfortunately, typical profiles of $K n_{e f f}$ in divertors and near limiters are found to be about one or larger in those regions, in which the dominant neutral particle plasma interaction takes place.

This has two computationally important consequences:

1. the neutral gas transport has to be described on a kinetic rather than on a fluid level

2. the kinetic (Boltzmann-) equation is linear.

A large literature exists on the linear transport theory and the methods of solution. Analytical methods are e.g. discussed in [5], an extensive review of particle simulation ("Monte Carlo"-) techniques can be found in $[6]$.

The linear kinetic transport equation can be written most conveniently for the collision density $\Psi$, with $\Psi=\frac{1}{\lambda} v f_{0}$ (loc. cit.) as:

$$
\Psi(x)=S(x)+\int d x^{\prime} \Psi\left(x^{\prime}\right) \cdot K\left(x^{\prime} \rightarrow x\right)
$$


Here $\mathrm{x}^{\prime}$ and $\mathrm{x}$ are the states $\left(\underline{r^{\prime}}, \underline{v^{\prime}}, i^{\prime}\right)$ and $(\underline{r}, \underline{v}, i)$, respectively, in phase space, at two successive collisions. $\mathrm{i}$ and $i^{\prime}$ are species indices. $\mathrm{S}$ is the once collided contribution from sources $\mathrm{Q}$, and the kernel $\mathrm{K}$ is usually decomposed into a collision- and a transport kernel, i.e. $\mathrm{C}$ and $\mathrm{T}$, where

$$
\begin{array}{r}
K\left(\underline{r}^{\prime}, \underline{v}^{\prime}, i^{\prime} \rightarrow \underline{r}, \underline{v}, i\right)= \\
C\left(\underline{r}^{\prime} ; \underline{v}^{\prime}, i^{\prime} \rightarrow \underline{v}, i\right) \cdot T\left(\underline{v}, i ; \underline{r}^{\prime} \rightarrow \underline{r}\right) .
\end{array}
$$

The kernel $\mathrm{C}$ is (excluding normalization) the conditional distribution for new co-ordinates $(\underline{v}, \mathrm{i})$ given a collision at position $\underline{r}^{\prime}$ and can be decomposed further into:

$$
C\left(\underline{r}^{\prime} ; \underline{v}^{\prime}, i^{\prime} \rightarrow \underline{v}, i\right)=\sum_{k} p_{k} C_{k}\left(\underline{r}^{\prime} ; \underline{v}^{\prime}, i^{\prime} \rightarrow \underline{v}, i\right)
$$

with

$$
p_{k}=\frac{\sigma_{k}}{\sum_{k} \sigma_{k}}
$$

with summation over the index $\mathrm{k}$ for the different types of collision processes under consideration and $p_{k}$ defined as the (conditional) probability for a collision to be of type k. $\sigma_{k}$ is the total inverse local mean free path (dimension: 1/length) for each collision type. The normalizing factor

$$
c_{k}\left(x^{\prime}\right)=\sum_{i} \int d \underline{v} C_{k}\left(\underline{r}^{\prime}, \underline{v}^{\prime}, i^{\prime} \rightarrow \underline{v}, i\right), \hat{C}_{k}=\frac{1}{c_{k}} C_{k}
$$

gives the mean number of secondaries for this collision process. The function $\hat{C}_{k}$ then is a conditional probability density.

The kernel $\mathrm{T}$ describes the free streaming motion of the particles between the collision events, and basically is determined by the total mean free path (or, equivalently, by the total macroscopic cross-section). T can be interpreted as the distribution density for the distance $l$ for a free flight starting from $\underline{r}^{\prime}$ to the next point of collision $\underline{r}=\underline{r}^{\prime}+l \cdot \underline{v} /|\underline{v}|$

Despite its simple physical content (namely: expressing particle conservation in phase space) this linear kinetic equation is algebraically extremely complex, and can be solved analytically or numerically only under often pathologically simplified assumption. A statistical simulation, however, accounting for the full complexity without any restriction, is straight forward.

With a procedure similar to the one explained above for the mean lifetime of an ash particle in the reactor burning core, a Markoff chain can again be constructed from the terms in this transport equation. One may for example use $\mathrm{S}$ as initial (birth) distribution and the kernel $\mathrm{K}$ as transition probability from

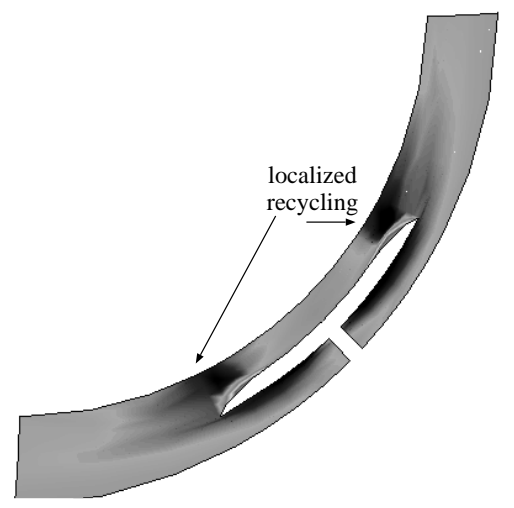

Figure 7: Re-ionization source distribution around ALT2 limiter (TEXTOR). Note: the main fraction is ionized within the confined plasma region (distinct from divertors)

one event to the next. Termination of a history can again be formulated in terms of the pumping probability, but in addition the ionization process provides a further, physically motivated, absorption probability. (Strictly speaking, the absorption probability used in the Markoff process needs not be related to a physical particle absorption process, but it facilitates interpretation of histories generated from the Markoff process, as e.g. in figures shown above.

Distinct from the simple Markoff chain discussed above, now the relevant macroscopic quantities (densities, re-ionization rates, pumped fluxes etc.) cannot be computed directly. Instead random number generators are employed to generate a large set (a few 10.000) of random walks. These are then processed into profiles of the required quantities by statistical averaging.

As outlined above the mean value of the time spend by all histories in any particular region of the plasma can directly be scaled into an estimate of the neutral particle density. Likewise, the spatially resolved reionization rate can be obtained by proper scaling of the statistical mean (over the random walks) of the ratio of path length to the ionization mean free path in any particular cell of the computational volume.

Note that this and related profiles enter as source terms in the fluid equations which are generally used for describing tokamak edge plasmas

Figure 7 shows one such re-ionization profile in the vicinity of the ALT-II pump-limiter in TEXTOR. Plasma and neutral gas transport are computed consis- 
tently by iteration a finite element plasma fluid code with a kinetic neutral particle ("Monte-Carlo-) code until overall convergence [7]. Even under such rather open limiter conditions (compared with high recycling divertors, in which the neutral particle cloud usually is much more localized, the neutral particle recycling terms are the dominant terms in the plasma fluid equations near the target surfaces and determine the plasma flow there. Typically the radial plasma flow near limiters is driven by localized neutral particle recycling. On top of the limiter, often the radial plasma flow is found to be reversed (back into the plasma core).

This feature is characteristic for limiters and one of the main arguments in favor of the technologically much more demanding divertor configuration. Given that collisional friction is the dominant force acting on surface released impurities and recycling helium particles, the risk of plasma poisoning and insufficient helium removal is obvious. A major effort in limiter tokamak studies, therefore, is devoted to this issue.

In principle, divertors do not suffer from such problems. Since the plasma flow is channelled outside (or at least far away from) the plasma core, direct contamination by surface released impurities is less likely. In particular the strong parallel plasma flow towards the target surface provides the impurity and helium retention mechanism.

However, as recent computer studies and also a few experiments have revealed, also these concepts can suffer from a certain kind of flow reversal: if the accumulated recycling sources within one flow channel are larger than the parallel plasma flow to the divertor target in this channel, the plasma flow in this channel has to reverse (as a simple consequence of particle conservation).

Such excess re-ionization can occur in some flow channels (at the expense of other flow channels) due to the unhindered cross field motion (via CX) of the neutral particles. The onset of this flow reversal depends sensitively on details of pumping and baffling in the divertor. If pumping is too inefficient, (either because of physical and engineering constrains, or on purpose because of the beneficial effects of large neutral gas densities in divertor plasma for heat dissipation), in some parts of the divertor plasma the plasma flow can reverse (away from the target, along the field-lines, back into the confined discharge). And, depending on the location of their sources, impurities and helium particles might be dragged back into the main discharge ("the divertor throws up").

\section{CONCLUSIONS}

The issue of helium removal for steady plasma burn is apparently very closely connected with the details of plasma recycling via the neutral particle channel. Firstly because only neutral ash particles can be channelled into pumping stations, but secondly also because of the effects of neutral particle recycling on the edge plasma flow, and thus on the forces acting on the ionized helium particles. Various conflicting requirements have to be met simultaneously such as:

good confinement (for energy balance)

poor confinement (for particle throughput)

strong pumping (for ash removal)

weak pumping (for the favorable high recycling conditions)

The search for a plasma edge configuration compatible with all these constrains, both experimentally and by computer simulations, is one of the key design issues to be solved before a reliable plasma surface interaction concept for ITER (and a future reactor) can be developed.

\section{REFERENCES}

1. E. REBHAN and G. VAN OOST, "Thermonuclear burn criteria for DT-plasmas", this volume.

2. D. REITER, G.H. WOLF, H. KEVER, "Burn Condition, Helium Particle Confinement and Exhaust Efficiency", Nucl. Fusion, 30 (1990), p2141

3. D. REITER, G.H. WOLF, H. KEVER, "Stationary burning: Analysis of profile effects", J. Nucl.Mater.,176\&177, (1990), p756

4. D. REITER et al., "Helium Removal from Tokamaks", Plasma Physics and Controlled Fusion, 33, 13, (1991), p1579

5. K.M. CASE, P.F. ZWEIFEL, "Linear transport theory",

Addison Wesley Pub. Comp., (1967)

6. J. SPANIER, E.M. GELBARD, "Monte-Carlo principles and neutron transport problems", Addison Wesley Pub. Comp., (1969)

7. D. REITER, "Neutral Gas Transport in Fusion Devices" in: Atomic and Plasma-Material Interaction Processes in Controlled Thermonuclear Fusion, R.K. JANEV and H.W. DRAWIN (eds.), Elsevier Science Publishers B.V., (1993)

8. D. REITER, "The EIRENE code users manual", http://www.eirene.de (2006) 